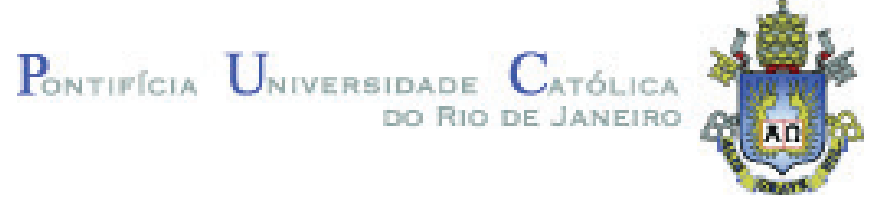

Artur Manoel Passos

\title{
Essays in Portfolio Management and Stocks Return Forecasting
}

M.A. Dissertation

Thesis presented to the Postgraduate Program in Economics of the Departamento de Economia, PUC-Rio as partial fulfillment of the requirements for the degree of Mestre em Economia

Advisor: Prof. Marcelo Medeiros

Rio de Janeiro

April 2011 


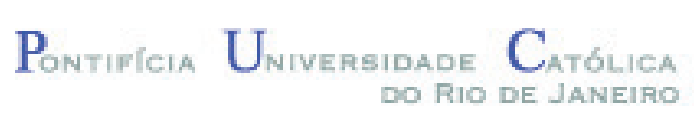

Artur Manoel Passos

\title{
Essays in Portfolio Management and Stocks Return Forecasting
}

Thesis presented to the Postgraduate Program in Economics of the Departamento de Economia, PUC-Rio as partial fulfillment of the requirements for the degree of Mestre em Economia. Approved by the following commission:

\author{
Prof. Marcelo Medeiros \\ Advisor \\ Departamento de Economia - PUC-Rio
}

Prof. Alexandre Lowenkron

Banco BBM

Prof. Caio Ibsen Rodrigues Almeida

FGV/EPGE

Prof. Mônica Herz

Coordinator of the Social Science Center - PUC-Rio

Rio de Janeiro - April 11, 2011 
All rights reserved.

\section{Artur Manoel Passos}

Artur Manoel Passos graduated in Computer Engineering at Unicamp in 2004. He worked as a Systems Architect for Spring Wireless from 2005 to 2008. He has attended the graduate program in Economics at PUC-Rio from 2009 to 2010. Currently, he works as an Economic Research Analyst for Itaú BBA.

Bibliographic data

Passos, Artur Manoel

Essays in Portfolio Management and Stocks Return Forecasting / Artur Manoel Passos ; advisor: Marcelo Medeiros. $-2011$.

$61 \mathrm{f}$ : : il. ; $30 \mathrm{~cm}$

Dissertação (Mestrado em Economia)-Pontifícia Universidade Católica do Rio de Janeiro, Rio de Janeiro, 2011.

Inclui bibliografia

1. Economia - Teses. 2. Gestão de Carteiras. 3. Otimização de Markowitz. 4. Previsão de Retornos de Ações. 5. Modelos de Múltiplos Fatores. 6. Estimação com Lasso. 7. Combinação de Modelos. I. Medeiros, Marcelo. II. Pontifícia Universidade Católica do Rio de Janeiro. Departamento de Economia. III. Título. 


\section{Acknowledgments}

Ao meu orientador Marcelo Medeiros, pelos ensinamentos, apoio e paciência.

A todos os professores e funcionários do Departamento de Economia da PUC-Rio, pelo apoio ao longo do curso e conhecimento transmitido. Em particular, ao professor Walter Novaes, por toda a teoria econômica e financeira que ensinou.

Aos amigos de minha antiga vida: Ivens, Leandro e Thiago, que me apoiaram a começar de novo.

A todos os amigos e colegas do programa de pós-graduação em Economia. Em particular, a Breno, Pablo e Rodrigo, pelo companheirismo, pela ajuda e conselhos que facilitaram tanto o curso quanto o que veio depois.

Ao amigo Rudi.

Aos meus pais Stalin e Ana Maria, pelo exemplo que deram em suas vidas, pela educação, e pelo amor incondicional.

Ao CAPES, ao CNPq e ao Departamento de Economia da PUC-Rio, pelos auxílios concedidos, sem os quais este trabalho não poderia ter sido realizado. 


\section{Abstract}

Passos, Artur Manoel; Medeiros, Marcelo (Advisor). Essays in Portfolio Management and Stocks Return Forecasting. Rio de Janeiro, 2011. 61p. M.A. Dissertation - Departamento de Economia, Pontifícia Universidade Católica do Rio de Janeiro.

The dissertation consists of three empirical essays which use historical data of stocks listed in NYSE. The first essay evaluates a portfolio selection approach based on the Markowitz optimization. Results show the portfolios have positive economic value, even after including transaction costs. The second essay compares the technique proposed in the first essay to the parametric approach. Results show the parametric approach performs worse than the modified Markowitz approach and shlightly better than the aggregated market. This suggests that exploring the covariance structure of stocks provides better results than overweighting stocks with characteristics associated to better riskreturn ratios in the past. The third essay evaluates models that forecast the cross-sectional variation in stock returns. Given the statistics used, benchmark models do not show greater forecasting power than skeptical or naive models. By using linear model combination or lasso technique on a model with several factors, I show it is possible to obtain slightly better results.

\section{Keywords}

Portfolio Management; Markowitz Optimization; Stock Return Forecasting; Multiple Factor Models; LASSO Estimation; Model Combination; 


\section{Resumo}

Passos, Artur Manoel; Medeiros, Marcelo (Orientador). Ensaios em Gestão de Carteiras e Previsão de Retornos de Ações. Rio de Janeiro, 2011. 61p. M.A. Dissertation — Departamento de Economia, Pontifícia Universidade Católica do Rio de Janeiro.

A dissertação é composta por três ensaios empíricos que usam dados históricos de ações americanas. O primeiro avalia o desempenho de uma abordagem de otimização de carteiras baseada na otimização de Markowitz. Os resultados mostram valor econômico positivo do portfólio resultante, mesmo na presença de custos de transação. O segundo artigo visa comparar e combinar a técnica desenvolvida no artigo anterior à abordagem paramétrica e avalia o desempenho da combinação das técnicas. Os resultados mostram que o desempenho da técnica paramétrica é inferior à técnica de Markowitz modificada e pouco melhor do que o mercado agregado. Isto sugere que o valor econômico de explorar a estrutura de covariância entre as ações é superior a aumentar pesos em ações cujas características oferecem relações risco-retorno maiores até o período. O terceiro ensaio avalia modelos de previsão da variação de retornos entre ações. As estatísticas utilizadas apontam que os modelos padrão não possuem poder preditivo superior a modelos que supõem que não há variação ou que usam a média histórica. Por meio do uso tanto de combinações de modelos lineares quanto estimação restrita de modelos com muitos fatores, mostro que é possível obter resultados ligeiramente superiores.

\section{Palavras-chave}

Gestão de Carteiras; Otimização de Markowitz; Previsão de Retornos de Ações; Modelos de Múltiplos Fatores; Estimação com Lasso; Combinação de Modelos; 


\section{Contents}

1 Evaluating an Adjusted Markowitz Approach on a Large Set of Stocks 10

$\begin{array}{lll}1.1 & \text { Introduction } & 10\end{array}$

1.2 Markowitz optimization and extensions 12

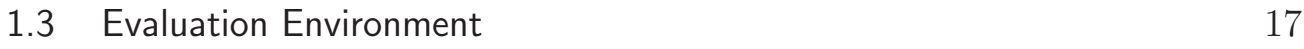

$\begin{array}{lll}1.4 & \text { Empirical results } & 20\end{array}$

$\begin{array}{lll}1.5 \text { Conclusions } & 27\end{array}$

2 An Empirical Comparison of Two Portfolio Selection Approaches 29

2.1 Introduction 29

2.2 Comparison Methodology 31

$\begin{array}{lll}2.3 & \text { Empirical results } & 35\end{array}$

2.4 Conclusions 44

3 Forecasting the cross-sectional variation in stock returns $\quad 46$

3.1 Introduction 46

3.2 Problem Formulation 47

3.3 Short-horizon statistics 48

3.4 Search for a better model $\quad 52$

3.5 Economic Value of each model $\quad 57$

$\begin{array}{lll}3.6 & \text { Conclusions } & 58\end{array}$

$\begin{array}{llr}4 & \text { References } & 59\end{array}$ 


\section{List of Figures}

1.1 Accumulated return for value-weighted and equal-weighted portfolios

2.1 Accumulated returns for value-weighted and equal-weighted portfolios 36

2.2 Sharpe Ratio for each year 


\section{List of Tables}

1.1 Correlation matrix 22

1.2 Naive and Markowitz portfolios performance 24

1.3 The economic value of Markowitz optimized portfolios 25

1.4 Naive and Markowitz portfolios performance segmented by bull and bear years 26

$\begin{array}{ll}1.5 & \text { Two regimes } \\ 1.6 & 27\end{array}$

1.6 Two regimes - results segmented by regime 28

2.1 Naive and parametric portfolios performance 39

2.2 Markowitz portfolios performance 40

2.3 Parametric technique deviating from alternate portfolios 41

2.4 Two regimes 43

2.5 Two regimes - positive slope regime 43

2.6 Two regimes - inverted slope regime 44

3.1 Cross-sectional statistics 49

3.2 Results - growing window of estimation $\quad 51$

3.3 Results - rolling window of estimation 52

3.4 Results - best equal-weighted combination of 7 models 54

3.5 Results - best equal-weighted combination of 7 models, out-ofsample procedure 54

3.6 Results - Performance of models estimated by Lasso 56

3.7 The economic value of Markowitz optimized portfolios 58 